\title{
Crystalline-State Configurations of Alkylene Sulfide Polymers*
}

\author{
William J. WELSH, ${ }^{* 1, * 2}$ James E. MARK, ${ }^{* 2}$ and Evaristo RIANDE*3 \\ *1 Department of Chemistry, Edgecliff College, Cincinnati, Ohio 45206, U.S.A. \\ ${ }^{* 2}$ Department of Chemistry and Polymer Research Center, \\ The University of Cincinnati, Cincinnati, Ohio 45221, U.S.A. \\ ${ }^{*}$ Instituto de Plásticos y Caucho, Madrid-6, Spain.
}

(Received February 29, 1980)

\begin{abstract}
Semi-empirical potential energy functions were used to calculate the conformational energies of several alkylene sulfide polymers of structure $\left[\mathrm{S}\left(\mathrm{CH}_{2}\right)_{y}\right]$. The polymers chosen were those for which crystalline-state configurations were known, specifically those having $y=1,2$, 3 , and 5 , respectively. The configurations of minimum conformational energy were generally found to correspond to the crystalline-state configurations. The only exceptions occurred for conformational-energy differences close to zero, which is to be expected since in these cases even small differences in intermolecular packing energies could obviously play an important role. Some preliminary comments are also made on the here-to-fore unstudied polysulfides corresponding to $y=4,6$, and higher. Comparisons with the corresponding polyoxides $\left.\left[\mathrm{O} \mathrm{CH}_{2}\right)_{y}\right]$ help elucidate the effect of the size of the hetero-atom X ( $\mathrm{S}$ or $\mathrm{O}$ ), its effective charge, the $\mathrm{C}-\mathrm{X}$ bond length, and the $\mathrm{C}-$ $\mathrm{X}-\mathrm{C}$ bond angle. The most important difference between the polysulfides and polyoxides appears to be the fact that the $\mathrm{C}-\mathrm{S}$ bond is considerably longer than the $\mathrm{C}-\mathrm{O}$ bond.
\end{abstract}

KEY WORDS Polysulfides / Polyoxides / Crystalline Polymers / Conformational Energies / Regular Configurations / Poly(methylene sulfide) / Poly(ethylene sulfide) / Poly(trimethylene sulfide) / Poly(pentamethylene sulfide) /

An understanding of the configurations of a polymer molecule requires a knowledge of the relative energies of the conformational states accessible to the skeletal bonds of the chain. ${ }^{1}$ Experimental values of these energies may be obtained from either spectroscopic studies of model compounds, or by comparisons between theoretical and experimental values of some configurationdependent properties of the polymer, usually in the random-coil state in solution. An alternative method involves the calculation of these energies from the various interatomic separations in the conformations of interest using standard semi-empirical potential-energy functions. Both approaches provide a bonus in that they identify the configuration of minimum (intramolecular) energy. This is of considerable interpretive and predictive value, since the minimum energy configuration is almost in-

* Presented in part at the IUPAC International Symposium on Macromolecules, Florence, September 712, 1980 . variably the one adopted by the chain in the crystalline state. ${ }^{1-3}$

The present study focuses on calculations of conformational energies of four alkylene sulfide polymers, the crystalline-state configurations of which are known from X-ray diffraction analyses. $^{4-7}$ Comparisons are carried out between minimum energy conformations and crystallinestate configurations for each of these polysulfides,${ }^{4-9}$ with some comparisons to the corresponding information on the analogous polyoxides $^{1,8,10-20}$ as well.

\section{THEORY}

\section{Structural Information}

Appropriate values of the bond lengths, bond angles $\theta$, and partial charges $q$ were obtained from studies of structurally related small molecules. ${ }^{8,9,21,22}$ Bond lengths for $\mathrm{C}-\mathrm{S}, \mathrm{C}-\mathrm{C}$, and C$\mathrm{H}$ bonds are $1.815,1.53$, and $1.09 \AA$, respectively. 
The very important $\mathrm{C}-\mathrm{S}$ skeletal bond is thus seen to have a length approximately $27 \%$ greater than that $(1.43 \AA)^{1,10,23,24}$ of the corresponding $\mathrm{C}-\mathrm{O}$ bond in the polyoxides. Values of the $\mathrm{C}-\mathrm{S}-\mathrm{C}, \mathrm{C}-\mathrm{C}-$ $\mathrm{S}, \mathrm{C}-\mathrm{C}-\mathrm{C}$, and $\mathrm{C}-\mathrm{C}-\mathrm{H}$ bond angles are 100,114 , 111.5 , and $110^{\circ}$, respectively. The $\mathrm{C}-\mathrm{S}-\mathrm{C}$ bond angle is thus approximately $12^{\circ}$ smaller than the corresponding $\mathrm{C}-\mathrm{O}-\mathrm{C}$ bond angle $\left(111.5^{\circ}\right)$ in a typical polyoxide. ${ }^{1,10,23,24}$ There does not seem to be a reliable value for the bond angle $\mathrm{S}-\mathrm{C}-\mathrm{S}$. It is probably reasonable to assume $\mathrm{e}^{4}$ that it has a value very close to that of $\mathrm{C}-\mathrm{C}-\mathrm{S}$, as the angle $\mathrm{O}-\mathrm{C}-\mathrm{O}$ $\left(112.0^{\circ}\right)$ has to that of $\mathrm{C}-\mathrm{C}-\mathrm{O}\left(111.5^{\circ}\right) .^{10}$ It was therefore tentatively assigned the value $114^{\circ}$ in the present investigation.

The $\mathrm{CH}_{2}-\mathrm{S}$ bond dipole moment is $1.21 \mathrm{D},{ }^{8,9,22}$ and this value, in conjunction with the $\mathrm{C}-\mathrm{S}$ bond length, indicates (i) a partial charge corresponding to -0.28 (of the electron charge) for an $\mathrm{S}$ atom, and +0.28 for a $\mathrm{CH}_{2}$ group between two $\mathrm{S}$ atoms, and (ii) +0.14 for a $\mathrm{CH}_{2}$ group between an $\mathrm{S}$ atom and another $\mathrm{CH}_{2}$ group. The $\mathrm{CH}_{2}-\mathrm{O}$ bond dipole moment is somewhat smaller $(1.07 \mathrm{D}),{ }^{8,10,22-24}$ but the significantly smaller $\mathrm{C}-\mathrm{O}$ bond length makes the corresponding partial charges slightly larger $(-0.31,+0.31$, and +0.155 , respectively).

\section{Calculation of Conformational Energies}

The energy of a given conformation of any of the polysulfide chains was taken to be the sum of van der Waals, torsional, and Coulombic contributions. ${ }^{1}$. The van der Waals interaction energy between atoms $i$ and $j$ separated by the distance $r_{i j}$ was calculated from the Buckingham potential function,

$$
E_{\mathrm{vdw}}=a_{i j} \exp \left(-b_{i j} r_{i j}\right)-c_{i j} / r_{i j}^{6}
$$

with the parameters $a, b$ and $c$ differing for different atom-pairs. The parameter $c$ in the attractive term was calculated from atomic polarizabilities $^{25}$ by application of the Slater-Kirkwood equation. ${ }^{26}$ The value of $b$ for a like atom-pair was taken from Scott and Scheraga, ${ }^{27}$ while the value for an unlike pair was given by the geometric mean $b_{i j}=\left(b_{i i} b_{j j}\right)^{1 / 2}$. The corresponding value of the parameter $a$ was then determined by minimizing eq 1 at $r=R_{\text {min }} \equiv R_{i}+R_{j}$, where $R_{i}$ and $R_{j}$ are the van der Waals radii of the interacting atoms. Values of $R_{i}$ and $R_{j}$, taken from crystal-structure data, ${ }^{28}$ were augmented by $0.1 \AA$ in order to compensate for the absence, in the present application, of longrange attractive forces that are operative in the crystal. ${ }^{1}$ The resulting values of $R_{\min }, a, b$, and $c$ are given in Table I; included for purposes of comparison is the corresponding information for interactions involving $\mathrm{O}$ atoms.

The torsional contributions were represented by ${ }^{1,9}$

$$
E_{\text {tor }}=\left(E_{0} / 2\right)(1-\cos 3 \phi)
$$

where $E_{0}$ is the intrinsic barrier height and $\phi$ is the skeletal-bond rotational angle. This dihedral angle was taken to be zero in the state shown in Figure 1, with positive values of $\phi$ corresponding to the rotations required to advance a right-handed screw. Values of $E_{0}$ were chosen so as to reproduce the observed barrier heights when used in conjunction with the van der Waals contributions calculated as described above. For the $\mathrm{C}-\mathrm{C}, \mathrm{S}-\mathrm{C}$, and $\mathrm{O}-\mathrm{C}$ skeletal bonds, these observed barrier heights are

Table I. Parameters for the Buckingham potential functions

\begin{tabular}{ccccc}
\hline Atom-pair & $R_{\min }$ & $10^{-3} a^{\mathrm{a}}$ & $b^{\mathrm{a}}$ & $c^{\mathrm{a}}$ \\
\hline $\mathrm{C} \cdots \mathrm{C}$ & 3.6 & 908.6 & 4.59 & 363.0 \\
$\mathrm{C} \cdots \mathrm{H}$ & 3.1 & 86.1 & 4.57 & 127.0 \\
$\mathrm{H} \cdots \mathrm{H}$ & 2.6 & 9.95 & 4.54 & 45.2 \\
& & & & \\
$\mathrm{~S} \cdots \mathrm{S}$ & 3.8 & 1354. & 3.90 & 3688. \\
$\mathrm{~S} \cdots \mathrm{C}$ & 3.7 & 1162. & 4.25 & 1158. \\
$\mathrm{~S} \cdots \mathrm{H}$ & 3.2 & 123.2 & 4.22 & 407.0 \\
$\mathrm{O} \cdots \mathrm{O}$ & 3.2 & 223.0 & 4.59 & 245.0 \\
$\mathrm{O} \cdots \mathrm{C}$ & 3.4 & 438.5 & 4.59 & 294.0 \\
$\mathrm{O} \cdots \mathrm{H}$ & 2.9 & 41.9 & 4.57 & 96.5 \\
\hline
\end{tabular}

${ }^{\text {a }}$ Units are such as to give $E$ in $\mathrm{kcal} \mathrm{mol}^{-1}$ when $r$ is in $\AA$.

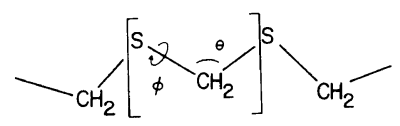

Figure 1. Schematic of the poly(methylene sulfide) (PMS) chain, showing the definition of one of the bond angles $\theta$ and one of the rotational angles $\phi$. The chain is arbitrarily shown in the all-trans conformation, and the brackets set off the chain repeat unit. 
3.00 (ethane), ${ }^{1} 2.13$ (dimethyl sulfide), ${ }^{9,29}$ and 2.72 $\mathrm{kcal} \mathrm{mol}{ }^{-1}$ (dimethyl ether). ${ }^{10}$ The resulting values of $E_{0}$ are $2.80,1.76$, and $1.80 \mathrm{kcal} \mathrm{mol}^{-1}$, respectively.

The Coulombic contributions were calculated from

$$
E_{\text {coul }}=k q_{i} q_{j} / \varepsilon r_{i j}
$$

where $k=332.1$ is a conversion factor giving $E_{\text {coul }}$ in $\mathrm{kcal} \mathrm{mol}^{-1}$ when $q_{i}$ and $q_{j}$ are in fractional parts of the electron charge. The dielectric constant $\varepsilon$ was taken to be 3.0 , as is generally done in calculations of this type. ${ }^{1,10}$

The potential energy functions thus obtained gave values of the (total) conformational energy in agreement with several previous results ${ }^{8-10}$ used for testing purposes. In the present investigation, they were used to calculate conformational energies of poly(methylene sulfide) (PMS), poly(ethylene sulfide) (PES), poly(trimethylene sulfide) (P3MS), and poly(pentamethylene sulfide) (P5MS). Different conformations were generated by rotations $\phi$ about the polysulfide skeletal bonds. Interest centered, of course, on conformations near the usual trans $(\mathrm{t})$, gauche positive $\left(\mathrm{g}^{+}\right)$, and gauche negative $\left(\mathrm{g}^{-}\right)$states located at $\phi=0^{\circ}, 120^{\circ}$, and $-120^{\circ}$, respectively. ${ }^{1,9,10}$ Distances $r_{i j}$ between pairs of atoms separated by 3 or 4 bonds were calculated in the usual manner, ${ }^{1}$ using geometric transformation matrices defined about the chain bonds. Rotational angles about a particular skeletal bond were varied by intervals of $5^{\circ}$, followed by closer scans at intervals of $1^{\circ}$ near the regions of low energy.

\section{RESULTS AND DISCUSSION}

The results most relevant to the crystalline-state configurations are the relative probabilities of the conformational states, and thus the energy differences $\Delta E$ between gauche and trans states. This information is summarized in Table II, and is discussed in detail separately for each of the four

Table II. Locations and energies of gauche states in polysulfides and polyoxides

\begin{tabular}{|c|c|c|c|c|c|c|}
\hline \multirow{2}{*}{ Polymer } & \multirow{2}{*}{$\begin{array}{c}\text { Bond } \\
\text { number }\end{array}$} & \multirow{2}{*}{ Interaction } & \multirow{2}{*}{$\frac{|\phi|}{\operatorname{deg}}$} & \multirow{2}{*}{$\frac{\Delta E^{\mathrm{b}}}{\mathrm{kcal} \mathrm{mol}^{-1}}$} & \multirow{2}{*}{$\frac{d^{\mathrm{c}}}{\AA}$} & \multirow{2}{*}{$\frac{R_{i}+R_{j}^{\mathrm{d}}}{\AA}$} \\
\hline & & & & & & \\
\hline PMS & 1 or 2 & $\mathrm{CH}_{2} \cdots \mathrm{S}$ & 114.0 & -0.63 & 3.43 & 3.9 \\
\hline PMO & 1 or 2 & $\mathrm{CH}_{2} \cdots \mathrm{O}$ & 115.0 & -0.34 & 2.84 & 3.6 \\
\hline PES & 1 & $\mathrm{CH}_{2} \cdots \mathrm{CH}_{2}$ & 109.0 & 0.25 & 3.33 & 4.0 \\
\hline PEO & 1 & $\mathrm{CH}_{2} \cdots \mathrm{CH}_{2}$ & 98.5 & 1.37 & 3.09 & 4.0 \\
\hline PES & 2 & $s \cdots s$ & 113.0 & 0.71 & 3.52 & 3.8 \\
\hline PEO & 2 & $\mathrm{O} \cdots \mathrm{O}$ & 116.5 & 0.82 & 2.93 & 3.2 \\
\hline P3MS & 1 & $\mathrm{CH}_{2} \cdots \mathrm{CH}_{2}$ & 109.5 & 0.11 & 3.33 & 4.0 \\
\hline P3MO & 1 & $\mathrm{CH}_{2} \cdots \mathrm{CH}_{2}$ & 99.0 & 1.22 & 3.09 & 4.0 \\
\hline P3MS & 2 & $\mathrm{~S} \cdots \mathrm{CH}_{2}$ & 113.0 & 0.09 & 3.31 & 3.9 \\
\hline $\mathrm{P} 3 \mathrm{MO}$ & 2 & $\mathrm{O} \cdots \mathrm{CH}_{2}$ & 118.5 & -0.24 & 2.97 & 3.6 \\
\hline P5MS & 2 & $\mathrm{~S} \cdots \mathrm{CH}_{2}$ & 112.5 & 0.36 & 3.31 & 3.9 \\
\hline $\mathrm{P} 5 \mathrm{MO}$ & 2 & $\mathrm{O} \cdots \mathrm{CH}_{2}$ & 117.5 & 0.15 & 2.98 & 3.6 \\
\hline $\left.\begin{array}{l}\text { P5MS } \\
\text { P5MO }\end{array}\right\}$ & 3 & $\mathrm{CH}_{2} \cdots \mathrm{CH}_{2}$ & 109.0 & 0.75 & 3.12 & 4.0 \\
\hline
\end{tabular}

a Number, reading from left to right, within repeat units shown in Figures $1-4$.

b Energy of the gauche ${ }^{ \pm}$state relative to trans.

c Distance between interacting groups in the gauche $e^{ \pm}$state.

d Sum of the van der Waals radii of the interacting groups. 
polysulfides and the corresponding polyoxides.

\section{Poly(methylene sulfide)}

According to the present calculations, the two skeletal bonds in the PMS repeat unit, shown in Figure 1, should have energies in the gauche states $\left(|\phi| \simeq 114^{\circ}\right)$ approximately $0.6 \mathrm{kcal} \mathrm{mol}^{-1}$ below that for the alternative trans state $\left(\phi=0^{\circ}\right)$. Poly(methylene oxide) (PMO) also has a calculated preference for gauche states, but with a smaller $\Delta E$ (approximately $-0.3 \mathrm{kcal} \mathrm{mol}^{-1}$ ). This difference between PMS and PMO is due to the relatively large steric repulsions occurring in PMO because of the very short $\mathrm{C}-\mathrm{O}$ bonds. As can be seen in the last two columns in Table II, the interacting groups in the gauche state in PMO are $0.8 \AA$ below the sum of the van der Waals radii, whereas they are only $0.5 \AA$ below in the case of PMS. It should be mentioned, however, that the observed value of $\Delta E$ in PMO is approximately $-1.4 \mathrm{kcal} \mathrm{mol}^{-1},{ }^{10}$ which is considerably underestimated by the calculated value, $-0.3 \mathrm{kcal} \mathrm{mol}{ }^{-1} \cdot{ }^{10}$ This difference is called a "gauche effect," 30 and a similar discrepancy may occur in the case of PMS. On the basis of comparisons between theoretical and experimental values of some configuration-dependent properties of poly(propylene sulfide), ${ }^{9}$ however, it appears that this type of discrepancy may generally be considerably smaller in the polysulfides.

In any case, gauche states are strongly preferred in both PMS and PMO. As a result, $\mathrm{g}^{ \pm} \mathrm{g}^{\mp}$ conformational pairs would not be as strongly suppressed by "pentane-type interferences" as they are in other chain molecules. ${ }^{1}$ Relatively low energies for $\mathrm{g}^{ \pm} \mathrm{g}^{\mp}$ states means, of course, that such conformations must be taken into account in rotational isomeric state calculations of configuration-dependent properties. They would not be expected to occur in the crystalline-state configurations, however, even if they represented minimum energy conformations. These $\mathrm{g}^{ \pm} \mathrm{g}^{\mp}$ states correspond to abrupt reversals of chain direction, and this apparently can not be tolerated in the usual mode of polymer crystallization, in which the chain propagates along a rectilinear axis through a very large number of unit cells. (These circumstances apply to a wide variety of crystalline polymers, including the fibrous proteins. It would not, however, be expected to apply to globular proteins in the crystalline state, in which the occupants at the lattice sites are entire molecules. ${ }^{31}$ Such collapsed chains do indeed have numerous bends, turns, and reversals that would not be permitted in the case of chains crystallizing along an axis. $)^{31}$

With regard to the prediction of crystalline-state configurations, there does seem to be good agreement between theory and experiment in the case of PMS. The polymer crystallizes as a 17,9 helix, ${ }^{4}$ which is very similar to the POM $9_{5}$ helix (which does correspond to a sequence of approximately gauche states of the same sign). ${ }^{1,10}$

\section{Poly (ethylene sulfide)}

As shown in Figure 2, the repeat unit of the PES chain has three skeletal bonds. The first and last are calculated to have gauche states approximately 0.2 $\mathrm{kcal} \mathrm{mol}^{-1}$ above the trans. This value of $\Delta E$ is a few tenths of a kcal mol ${ }^{-1}$ higher than the value recently calculated by Abe, ${ }^{8,9}$ possibly because the two calculations are based on different PES sequence lengths. The agreement is thus satisfactory, considering the uncertainty in general in such calculated results. ${ }^{1}$ As shown in Table II, the two corresponding $\mathrm{C}-\mathrm{O}$ bonds in poly(ethylene oxide) (PEO) are much more constrained to the trans state. This is due to the very short length of these bonds and applies to the polyoxides in general. In any case, unfortunately, this energy difference in PES is not large enough to hazard a prediction for the conformation about this bond in the crystalline state since a small difference in intermolecular (packing) energy could be decisive in such cases. For the second bond, the trans state should definitely be preferred, the energy difference being estimated as $0.7 \mathrm{kcal} \mathrm{mol}{ }^{-1}$ in the present calculations, in reasonable agreement with the value $0.4 \mathrm{kcal} \mathrm{mol}^{-1}$ estimated earlier. ${ }^{9}$ The crystalline-state configuration was found to be $\left[\mathrm{g}^{ \pm} \mathrm{tg}^{ \pm}\right],{ }^{5}$ which is consistent with the above arguments. Crystalline PEO has the opposite configuration, $\left[\operatorname{tg}^{ \pm} t\right] .^{10,12,13}$ PEO also exhibits a crystalline modification corresponding to $\left[\mathrm{tt} t,{ }^{14}\right.$ and the above analysis suggests that this may

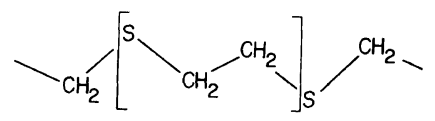

Figure 2. Schematic of the poly(ethylene sulfide) (PES) chain. 
be an alternative low-energy conformation for PES as well.

\section{Poly(trimethylene sulfide)}

There are four skeletal bonds in the P3MS repeat unit, as is illustrated in Figure 3, and all should have gauche and trans states of essentially the same energy (within $0.1 \mathrm{kcal} \mathrm{mol}^{-1}$ ). This molecule may therefore be an interesting approximation to the "freely-rotating" chain much used as a highly idealized reference state in configurational analyses. ${ }^{1}$ In analogy to the conclusion reached in the comparison between PES and PEO, P3MS seems to be a less constrained molecule than poly(trimethylene oxide) (P3MO).

The crystalline-state configuration of P3MS has been reported to be $\left[\mathrm{g}^{ \pm} \mathrm{g}^{ \pm} \mathrm{g}^{ \pm} \mathrm{g}^{ \pm}\right],{ }^{6}$ which is consistent with the present analysis. In contrast, P3MO exhibits three crystalline modifications, [tttt], $\left[\operatorname{tg}^{ \pm} t\right]$, and $\left[\operatorname{tg}^{ \pm} \mathrm{g}^{ \pm} \mathrm{t}\right] \cdot{ }^{12,16}$ In all of them, the $\mathrm{C}-\mathrm{O}$ bonds remain trans, as expected.

\section{Poly(pentamethylene sulfide)}

The P5MS repeat unit, shown in Figure 4, has six skeletal bonds, and the first and last of these should have gauche and trans states of essentially the same energy. In the case of the second and fifth bonds, trans states should be preferred by approximately $0.4 \mathrm{kcal} \mathrm{mol}^{-1}$; the same preference should exist for the third and fourth bonds, with an energy difference of approximately $0.7 \mathrm{kcal} \mathrm{mol}^{-1}$. The crystalline-state configuration corresponds to a sequence of all-trans conformations, ${ }^{7}$ in agreement with the above analysis. The corresponding poly-

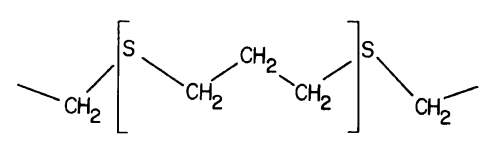

Figure 3. Schematic of the poly(trimethylene sulfide) (P3MS) chain.

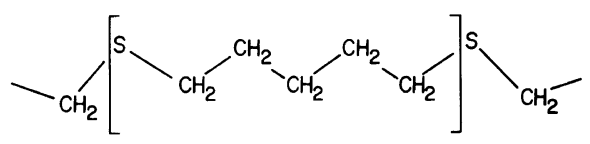

Figure 4. Schematic of the poly(pentamethylene sulfide) (P5MS) chain. oxide has apparently not been investigated in this regard.

\section{Other Alkylene Polysulfides}

The present calculations also permit some comments to be made on poly(tetramethylene sulfide), poly(hexamethylene sulfide), and the higher poly(alkylene sulfide)s as well. In general, the first and last bonds in these $\left[\mathrm{S}-\left(\mathrm{CH}_{2}\right)_{y}-\right]$ repeat units should have gauche and trans states of essentially the same energy, and the intervening bonds should prefer trans states. The longer the alkylene sequence, however, the more likely it is that the all-trans conformation will be the crystalline-state configuration. The more trans states there are in the repeat unit, the more difficult it would be to construct a helix having two gauche bonds per repeat unit that would compete in packing efficiency with the planar zig-zag, all-trans conformation.

\section{CONCLUSIONS}

The conformational energy calculations carried out are seen to be very useful for the interpretation and even prediction of the crystalline-state configurations of the poly(alkylene sulfide)s. They can also be utilized, of course, in rotational isomeric state calculations of the configuration-dependent properties of this very interesting class of chain molecules.

An additional important conclusion is that the polysulfides in general seem to have more configurational versatility than the corresponding polyoxides, primarily because of the relatively large value of the C-S bond length. They should thus be considered more flexible in the sense of generally having fewer skeletal bonds highly constrained to particular rotational states. The same conclusion was reached by $\mathrm{Abe}^{8,9}$ on the basis of calculations of conformational energies and configurational partition functions of PES and poly(propylene sulfide). It is also in agreement with the results of a study of the flexibilizing effect of atomic "swivels" introduced between rigid-rod sequences in aromatic heterocyclic polymers, in order to make them more tractable. Conformational-energy calculations carried out on these systems indicated that $\mathrm{S}$ swivels should be better than $\mathrm{O}$ swivels in imparting chain flexibility of this type. ${ }^{32}$ 
Acknowledgements. It is a pleasure to acknowledge the financial support provided by the National Science Foundation (Grant DMR 79-18903, Polymers Program, Division of Materials Research) and by the Air Force Office of Scientific Research (Grant AFOSR 78-3683, Chemical Structures Program, Division of Chemical Sciences), and the Fellowship from the Comision Asesora de Investigación Científica y Téchnica, which permitted E.R. to spend several months at the University of Cincinnati. We also wish to acknowledge, with gratitude, the very useful information and help provided by Professor A. Abe, Professor $\mathrm{H}$. Tadokoro, and Dr. Y. Takahashi.

\section{REFERENCES}

1. P. J. Flory, "Statistical Mechanics of Chain Molecules," Interscience, New York, N.Y., 1969.

2. J. E. Mark, J. Polym. Sci., C, 54, 91 (1976).

3. J. E. Mark, Acc. Chem. Res., 12, 49 (1979).

4. G. Carazzolo and G. Valle, Makromol. Chem., 90, 66 (1966).

5. Y. Takahashi, H. Tadokoro, and Y. Chatani, J. Macromol. Sci., Phys., B2, 361 (1968).

6. H. Sakakihara, Y. Takahashi, and H. Tadokoro, Preprints, SPSJ Symposium on Macromolecules, The Society of Polymer Science, Japan, Tokyo, November 11-13, 1969, 11 B11, p 407.

7 Y. Gotoh, H. Sakakihara, and H. Tadokoro, Polym. J., 4, 68 (1973).

8. A. Abe, Polym. Prepr. Am. Chem. Soc. Div. Polym. Chem., 20 (No. 1), 460 (1979).

9. A. Abe, Macromolecules, 13, (1980) in press.

10. A. Abe and J. E. Mark, J. Am. Chem. Soc., 98, 6468 (1976).

11. J. E. Mark, Macromolecules, 11, 627 (1978).

12. H. Tadokoro, "Structure of Crystalline Polymers,"
Interscience, New York, N.Y., 1979.

13. Y. Takahashi and H. Tadokoro, Macromolecules, 6 , 672 (1973).

14. Y. Takahashi, I. Sumita, and H. Tadokoro, J. Polym. Sci., Polym. Phys. Ed., 11, 2113 (1973).

15. J. E. Mark, J. Polym. Sci., B, 4, 825 (1966).

16. H. Tadokoro, Y. Takahashi, Y. Chatani, and H. Kakida, Makromol. Chem., 109, 96 (1967).

17. Y. Takahashi and J. E. Mark, J. Am. Chem. Soc., 98, 3756 (1976).

18. D. S. Chiu, Y. Takahashi, and J. E. Mark, Polymer, 17, 670 (1976).

19. J. E. Mark and D. S. Chiu, J. Chem. Phys., 66, 1901 (1977).

20. J. E. Mark, J. Chem. Phys., 67, 3300 (1977).

21. H. J. M. Bowen and L. E. Sutton, "Tables of Interatomic Distances and Configuration in Molecules and Ions," The Chemical Society, London, 1958; Supplement, 1965.

22. A. L. McClellan, "Tables of Experimental Dipole Moments," Vol. 1, W. H. Freeman, San Francisco, 1963.

23. A. Abe, T. Hirano, and T. Tsuruta, Macromolecules, 12, 1092 (1979).

24. A. Abe, T. Hirano, K. Tsuji, and T. Tsuruta, Macromolecules, 12, 1100 (1979).

25. J. A. A. Ketelaar, "Chemical Constitution," Elsevier Publishing Co., New York, N.Y., 1958.

26. K. S. Pitzer, Adv. Chem. Phys., 2, 59 (1959).

27. R. A. Scott and H. A. Scheraga, J. Chem. Phys., 42, 2209 (1965); ibid., 45, 2091 (1966).

28. A. Bondi, J. Phys. Chem., 68, 441 (1964).

29. L. Pierce and M. Hayashi, J. Chem. Phys., 35, 479 (1961).

30. S. Wolfe, Acc. Chem. Res., 5, 102 (1972).

31. C. Tanford, "Physical Chemistry of Macromolecules," Wiley, New York, N.Y., 1961.

32. W. J. Welsh, D. Bhaumik, and J. E. Mark, unpublished results. 